\title{
Characteristics of Ground Effects of an Unmanned Aerial Vehicle with Medium-aspect-ratio Flying Wings during Take-Off
}

\author{
Junli WANG*, Wensheng ZHANG*, Bolin FENG*, Zhigui REN*, Qinghe ZHAO** \\ *School of Mechanical Engineering, Shaanxi University of Technology, Hanzhong Shaanxi 723001, China, \\ E-mail:wjl503; @126.com; zws8211@163.com;18091604285@163.com; zhiguiren@163.com \\ **School of Energy and Power Engineering, Beihang University, Beijing 100191, China, E-mail: zhiguiren@163.com \\ cross $^{\text {ref }}$ http://dx.doi.org/10.5755/j01.mech.24.5.20934
}

\section{Nomenclature}

$h$-height of trailing edge above the ground; $c$ - chord length of the airfoil; $c_{A}-$ chord length of the wing root; $\lambda$ - aspect ratio; $b$-wing span; $C_{d}-$ drag coefficient; $C_{d}$ - lift coefficient; $C_{m}$ - pitching-moment coefficient; $C_{p}$ - pressure coefficient; $\alpha$ - angle of attack; $U$ - take-off velocity; $R e$ - Reynolds number

\section{Introduction}

With the continuous development of large and heavy-duty unmanned aerial vehicles (UAVs), take-off and landing technology has become a key link restricting its development. In the analysis of take-off and landing performances of UAVs, ground effect is one of the factors that have to be taken into consideration $[1,2]$. Ground effect refers to the nonlinear complex flow field relationships between the aircraft, the atmosphere, and the ground surface during take-off and landing. The large scale of UAVs with medium-aspect-ratio flying wing configurations strengthens the interaction of UAVs with the ground when taking off: this influences the longitudinal aerodynamic characteristics of UAVs. When the take-off height of UAVs with flying wing configurations is different, the ground effects vary correspondingly, so elevator deflection and take-off velocity of UAVs have to be adjusted according to the changes in ground effect. For UAVs with flying wing configurations that are difficult to be controlled in the longitudinal direction, ground effects exert significant influences on manipulation design for safe take-off, therefore, in view of UAVs with medium-aspect-ratio flying wing configurations, it is necessary to study the characteristics of ground effect during take-off and find accurate relationships between longitudinal aerodynamic characteristics with height from the ground, take-off velocity, and angle of attack.

Over the past few decades, scholars have studied ground effects by using experimental and numerical simulation methods. By fixing ground boundary conditions and moving ground boundary conditions, the characteristics of ground effects of symmetrical and asymmetrical aerofoil were researched, thus obtaining the influence of height from the ground and angle of attack in take-off on ground characteristics of aerofoil [3-6]. The ground effects of wings with low- and medium-aspect-ratios and a small sweepback angle were investigated [7-11]. The ground effects of largeswept delta wings were also studied under different angles of attack [12-15].

In recent years, the research on ground effects of UAVs has attracted the attention of many scholars. Jones investigated V-shaped tailless UAVs with a sweepback angle of $45^{\circ}$ on the leading edge and found that ground effects rise pitching moment and slope of lift curve and reduces induced drag, while the total drag on the UAV increases [16]. Elsewhere In the ground effects of V-and W-shaped UAVs were studied by using the experimental method [17]. Mustachio studied ground effects of unmanned combat aerial vehicles (UCAVs) with a W-shaped configuration and investigated the influences of aileron deflection [18]. Yang researched the impacts of asymmetric ground effects on lateral- and heading-directional aerodynamic characteristics of UAVs [19].

Based on the above research, ground effect of UAVs were mainly researched in the experiment by using moving ground to replace the original fixed ground boundary conditions. Although such a research method overcame the deficiency of low accuracy of experimentation when using fixed ground, the experimental costs and complexity were greatly increased. The low-aspect-ratio UAVs were mainly studied in numerical simulation. The take-off process only considered horizontal movement of the ground, while the influences of take-off velocity, take-off angle of attack, and height from the ground were not adequately taken into account. The aim of the study is to research characteristics of ground effects when UAVs with medium-aspect-ratio flying wings take-off to a certain height by using a numerical method. Moreover, the influence of ground effects under different angles of attack, heights from the ground, and take-off velocity on the aerodynamic characteristics of UAVs were investigated, which provides certain aerodynamic data useful for designing control systems governing the take-off process of medium-aspect-ratio UAVs.

\section{Methodology}

\subsection{Modelling of ground effects}

In the simulation of ground effects in take-off process of an UAV, the calculation model is as shown in Fig. 1. The influences of the ground on flow fields of the UAV need to be taken into account and the direction of free flows velocity should be parallel to the ground in any such calculation. For the above reason, the angle of attack does not show changes in free flows direction, but indicates the angle of the central axis of the UAV to the ground. The simulation of ground effects is based on the principles of relative motion. It is assumed that the UAV is motionless and the velocity of ground movement equals the take-off velocity $U_{\infty}$, which is realised by moving-ground boundary conditions. The height $h$ from the ground is defined as the height of the 
trailing edge of the fuselage above the ground and equals the product of mesh motion $U_{z}$ and the time since take-off, as realised by way of a dynamic meshing technique.

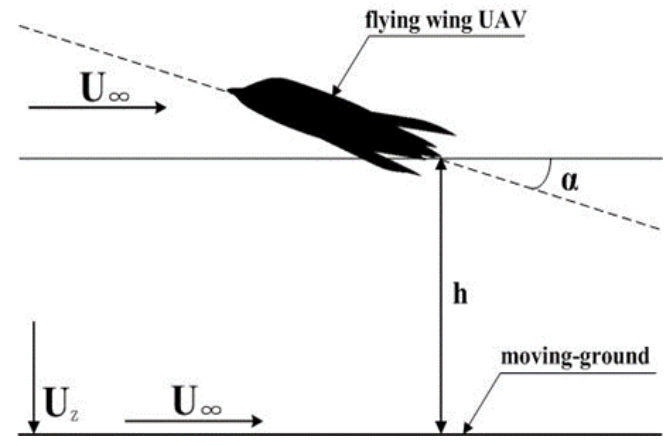

Fig. 1 The calculation model of ground effect

\subsection{Dynamic grid technology}

In the analysis of ground effects, with the increase of height from the ground of the UAV, the grid between the ground and the UAV extends from the initial small distance to a few meters or more. Therefore, the generation of the dynamic mesh is a key problem. If a dynamic mesh has poor robustness, it may result in negative grid elements, thus causing a failure during calculation. In the meantime, in the simulation of ground effects, the scale of grids near the ground cannot too be large; otherwise the flows in ground boundary layers and disturbance of ground boundary layers to flow fields of the UAV cannot be simulated accurately. In the study, structural grids and a dynamic layering technique were used to update the dynamic mesh used in the simulation. The core idea is to add dynamic layers according to the height of ground grid layers adjacent to the boundary to the motion (Fig. 2).

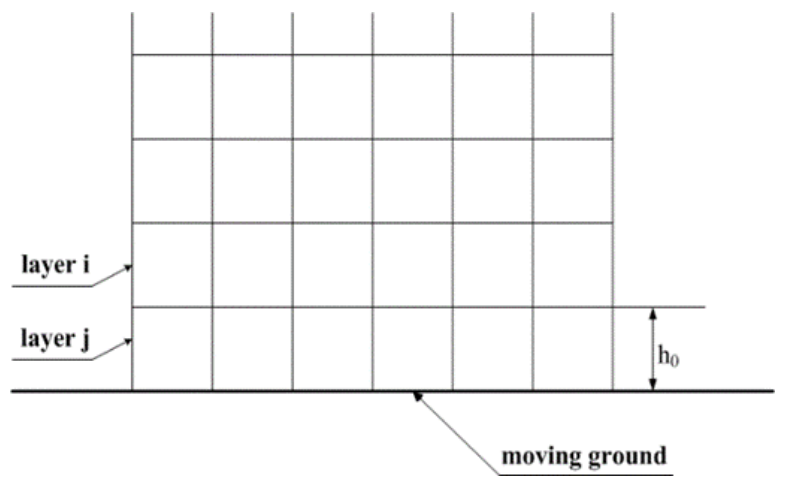

Fig. 2 The mesh model of dynamic layers

In Fig. 2, the grids in the jth layer are close to the motion boundary-ground, and $h_{0}$ indicates the height of grids in the jth layer. When the UAV took off, the height from the ground gradually increased and the grids in the jth layer were initially stretched. When the height of the grids increased to the critical value given by Formula (1), the grid elements in the jth layer were divided by using the constant ratio splitting method.

$$
h_{0 \min }>\left(1+a_{s}\right) h^{\prime}
$$

where: $h^{\prime}$ and $a_{s}$ indicate the height of ideal grid elements and the splitting factor of each layer.

\subsection{Governing equation}

The governing equation used is the unsteady Navier-Stokes equation and is expressed, in inertial Cartesian coordinates, as follows [20]:

$$
\frac{\partial}{\partial t} \iiint_{\Omega} \bar{Q} d \Omega+\iint_{S}\left(\bar{G}-\bar{Q}_{\vec{q}}\right) \cdot d \vec{S}=\frac{1}{R e} \iint_{S} \bar{F}^{V} \cdot d \vec{S},
$$

where: $\bar{Q}$ indicates the flow field variable, $\bar{G}, \bar{F}^{v}$, and $\vec{q}_{b}$ denote the convective term, the dissipation term, and the grid velocity, respectively. The equation is not in closedform. By introducing the thermodynamic relationship for a perfect gas and Stokes assumption, the above equation set forms closed-form equations. In solving the governing equations, the Spalart-Allmaras turbulence model was used and Roe flux-difference splitting scheme was used in space discretization [21]. Moreover, the fully implicit dual time stepping method was employed in time-marching, that is, implicit marching and explicit Runge-Kutta marching methods were used in real- and pseudo-time [22].

\section{Verification}

In order to verify the validity of the numerical method used in this paper, the wing in the literature [5] was calculated. The cross-sectional airfoil of the wing is NACA4412, and the chord length of the airfoil was $150 \mathrm{~mm}$ and the span of the wing was $600 \mathrm{~mm}$, as shown in Fig. 3.

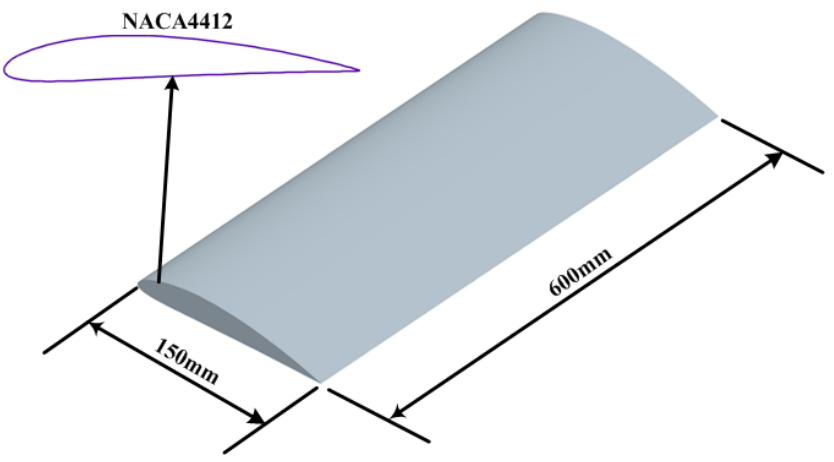

Fig. 3 Model of wing for CFD calculation

The parameters for calculation are shown as $U_{\infty}=30.8 \mathrm{~m} / \mathrm{s}, R e=3.0 \times 10^{5}$ and $\alpha=0^{0} 、 8^{0}$, along with moving ground boundary conditions. The calculation results of ground effects under different angles of attack and heights from the ground were compared with the test results in the literature [5], and comparisons of the results are shown in Figs. 4 and 5.

It can be seen from the above figures that the calculation results coincide with the test results, which indicates that the analysis method for ground effects proposed in the study is reliable.

\section{Results and discussions}

A UAV with $\mathrm{W}$-shaped flying wing configurations with zero sweep and torsion angle was adopted for analysis, as shown in Fig. 6. Moreover, it is found that the aspect ratio, span, and chord length of the wing root were $\lambda=11$, 


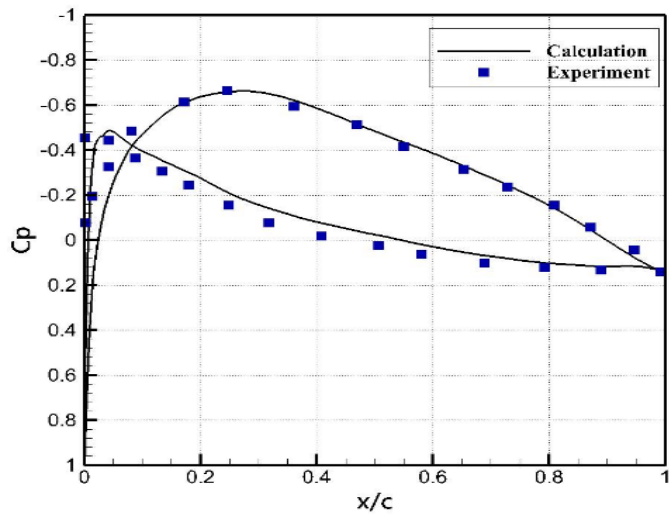

$\mathrm{a}$

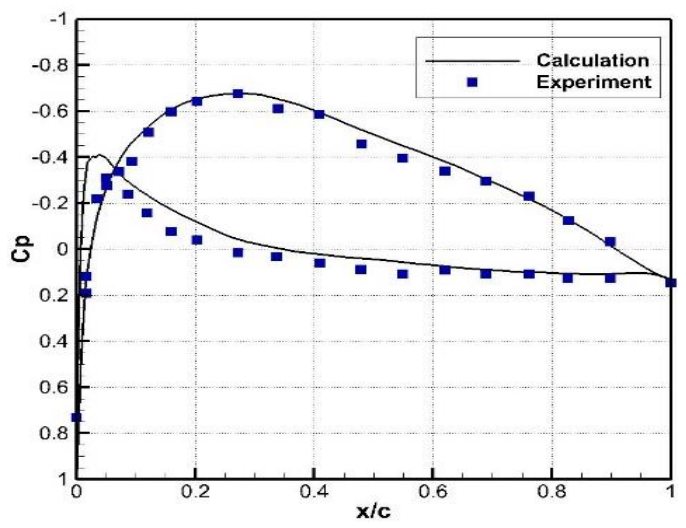

$\mathrm{b}$

Fig. 4 Wing mid-span pressure distribution for $\alpha=0^{0}$ : (a) $h / c=0.15 ;$ (b) $h / c=0.40$
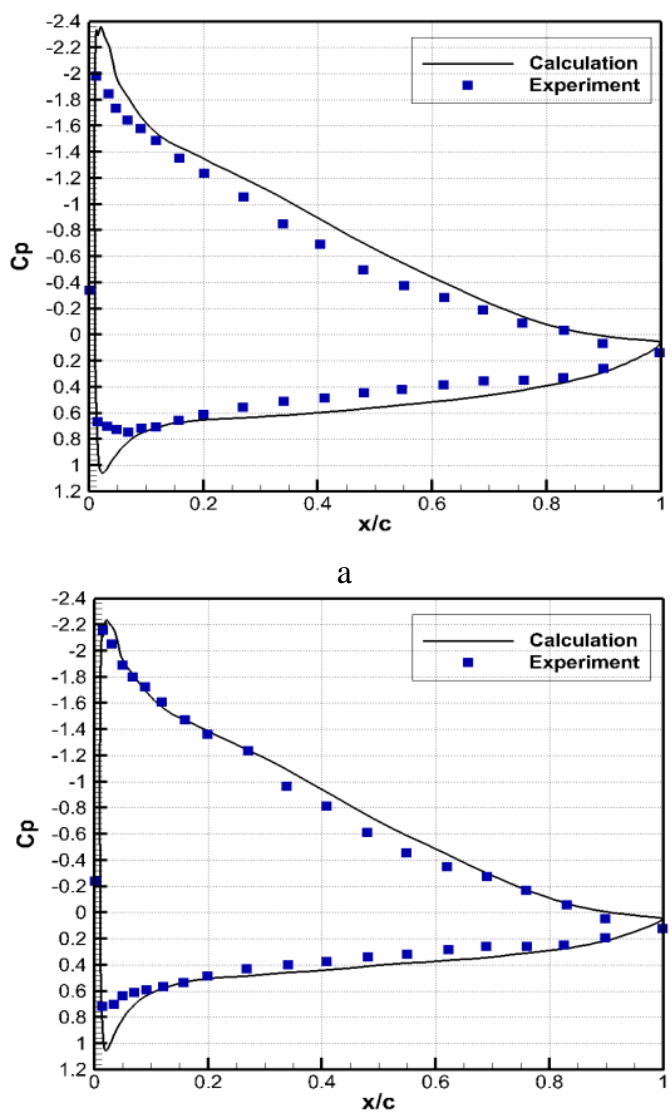

b

Fig. 5 Wing mid-span pressure distribution for $\alpha=8^{0}$ : (a) $h / c=0.15$; (b) $h / c=0.40$

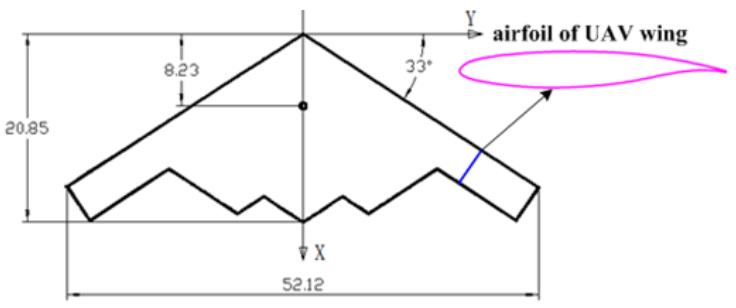

Fig. 6 Model of UAV for CFD calculation (Unit: m)

$b=52.12 \mathrm{~m}$, and $C_{A}=20.9 \mathrm{~m}$, respectively. The cross-section of the UAV wing is a thick airfoil with a curved edge at the trailing edge. Owing to it being the lift, drag, and longitudinal characteristics that were studied, a half-model of the UAV was analysed to reduce the computational burden. The coordinate origin was at the head of the UAV and the calculation was carried out in the domain which was 10 times the chord length of the wing root in the $\mathrm{X}$-direction and five times the half-length in the Y- and Z-directions. The structural grid, with its H-H topological structure, was used for grid division and the grids near wall surface and the ground were refined. The number of grid elements was $1.2 \times 10^{6}$. In the calculation, a half of the whole area being $245 \mathrm{~m}^{2}$ and the mean aerodynamic chord length being $12.94 \mathrm{~m}$ was taken for reference purposes. The distance from the reference point of pitching moment to the UAV head was $8.23 \mathrm{~m}$.

\subsection{Influence of take-off velocity}

According to the actual take-off velocity of the $\mathrm{UAV}$, three possible velocities were selected for calculation. Moreover, by setting the angle of attack as $\alpha=4^{0}$, the characteristics of ground effects of the UAV under different take-off velocities were obtained. The results are displayed in Fig. 7.

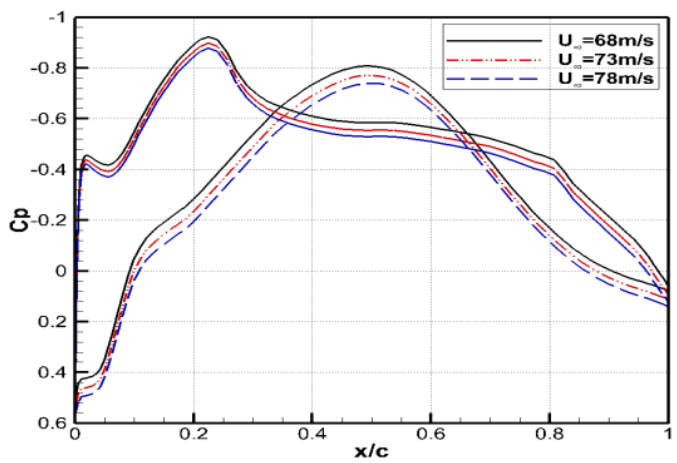

a

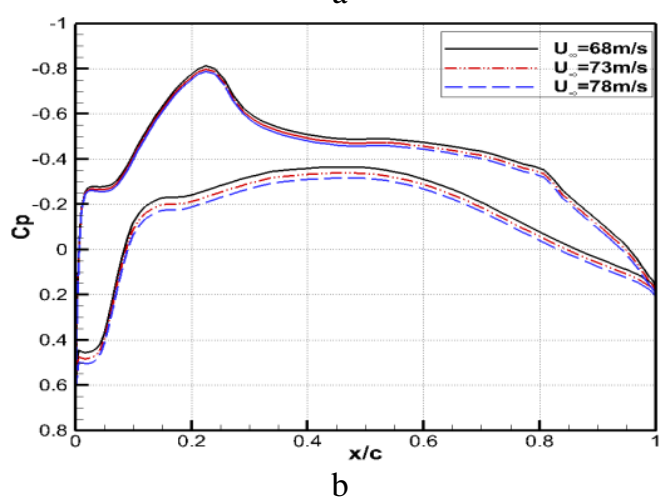

Fig. 7 Pressure coefficient distribution of model spanwise section at $y=2 \mathrm{~m}$ : (a) $h / c_{A}=0.067$; (b) $h / c_{A}=0.153$ 
As shown in the Fig. 7, with the increase in takeoff velocity, the suction area and amplitude of the lower surface of the UAV gradually decreased and the negative pressure on the upper surface decreased slightly.

\subsection{Influence of height from the ground}

When the angle of attack was set to $\alpha=2^{0}, 3^{0}, 4^{0}$, the characteristics of the ground effects of the UAV at different heights from the ground were obtained and the calculation results are shown in Fig. 8. A suction area was formed between the lower surface of the UAV and the ground and the suction force reached its peak value at $h / c_{A}=0.067$. When the height from the ground increased to $h / c_{A}=0.153$, the effects of air flow between the ground and the lower surface of the UAV were weakened and the peak suction force on the lower surface decreased. When the height from the ground reached $h / c_{A}=0.239$, the suction force on the lower surface of the UAV was quasi-stable and remained unchanged when the height from the ground increased further.

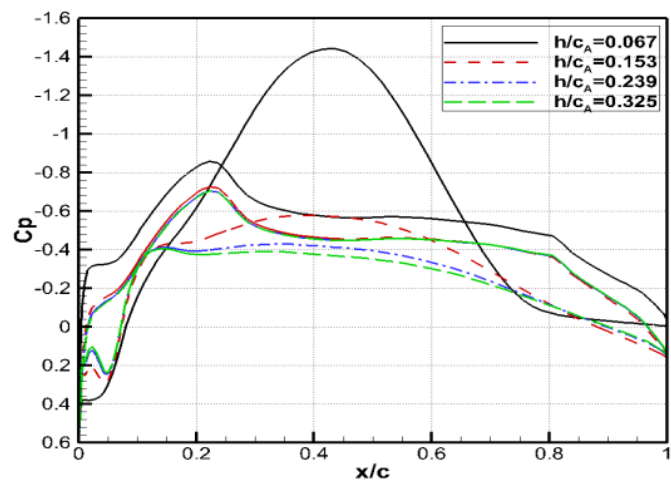

a

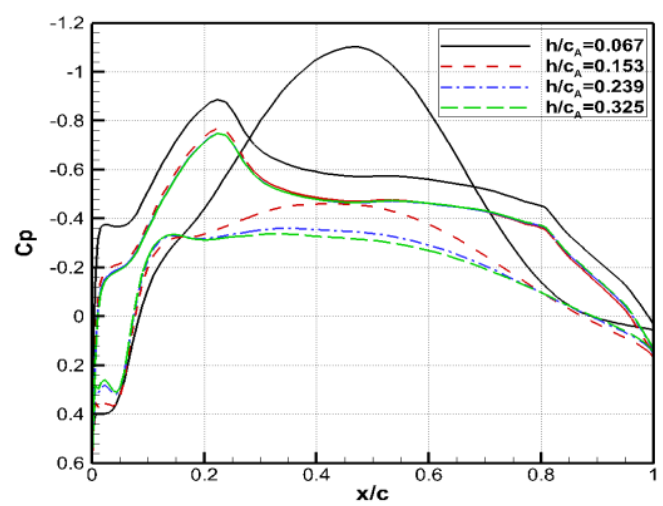

b

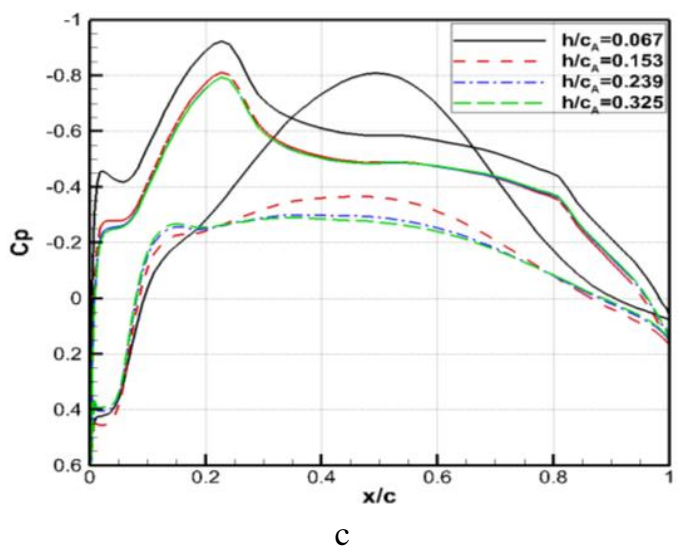

Fig. 8 Pressure coefficient distribution of model spanwise section at $y=2 \mathrm{~m}$ : (a) $\alpha=2^{0}$; (b) $\alpha=3^{0}$; (c) $\alpha=4^{0}$
It can be seen from the change curve of drag coefficient in Fig. 9, a that, with increased height from the ground, the effects of air flow between the ground and the UAV decreased. Therefore, the limitations of the ground on the down wash of wings of the UAV decreased, increasing the induced drag of the UAV, thus increasing its drag coefficient. When the height from the ground reached $h / c_{A}=0.5$, ground effects exerted only small influences on the aerodynamic characteristics of the UAV and drag coefficient remained unchanged even though the height from the ground increased further.

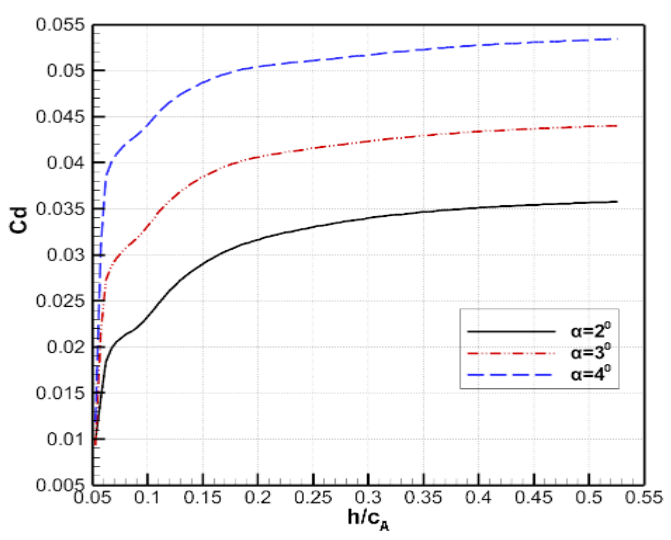

$\mathrm{a}$

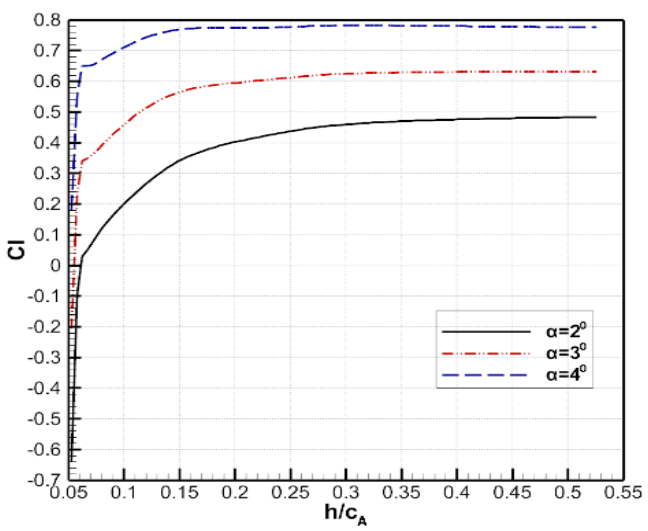

$\mathrm{b}$

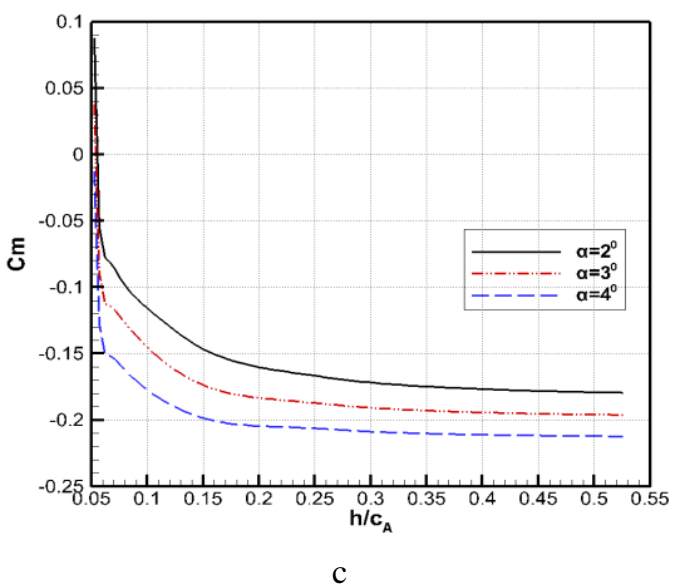

Fig. 9 Longitudinal aerodynamic characteristic of UAV: (a) drag coefficient; (b) lift coefficient; (c) pitching-moment coefficient

As demonstrated in Fig. 9, b, as the height from the ground decreased, the lift coefficient diminished to some 
small value due to the influences of the suction area developed on the lower surface of the wings. In particular, when the angle of attack was $2^{\circ}$, the suction amplitude on the lower surface of the wings was large and the lift coefficient was found to be a large negative value. With increasing height from the ground, the suction amplitude on the lower surface gradually decreased, while the lift coefficient gradually increased. When the height from the ground reached $h / c_{A}=0.5$, ground effects only slightly affected the aerodynamic characteristics of the UAV and the lift coefficient remained unchanged thereafter. As shown in Fig.9, c, at low altitudes, the pitching stability of the UAV decreased due to the influence of the suction area generated on the lower surface of the wings.

\subsection{Influence of take-off angle of attack}

By using the take-off velocity of $U_{\infty}=68 \mathrm{~m} / \mathrm{s}$, the characteristics of ground effects of the UAV under different angles of attack from the ground were obtained and the results are as shown in Fig. 10.

As demonstrated in Fig. 10, when the angle of attack was $2^{\circ}$, a small gap and a large flow velocity were shown between the lower surface and the ground, resulting in a large suction force on the lower surface. When the angle of attack gradually increased, the flow velocity between the lower surface and the ground gradually decreased and the peak suction force on the lower surface decreased.

\section{Conclusions}

The characteristics of ground effects on a UAV with medium-aspect-ratio flying wings in the take-off process were investigated from the perspective of the longitudinal aerodynamic characteristics. To study the influences of take-0ff velocity, the height from the ground, and the angle of attack, numerical simulations were conducted under different conditions. The conclusion is as follows:

1. The height from the ground exerted significant influences on the longitudinal aerodynamic characteristics of a medium-aspect-ratio UAV. When the height from the ground was small, a large suction area was formed on the lower surface of the wings. The suction amplitude increased by $37 \%$ with a decrease in the angle of attack, which resulted in a negative lift coefficient when the angle of attack was small. As the height of the ground increases, the suction area and amplitude of the lower surface of the wing decrease, and the lift coefficient increases. After the height from the ground reached $h / c_{A}=0.5$, ground effects exerted little influences on the UAV and the lift coefficient remained unchanged.

2. The angle of attack exerted a certain influence on the longitudinal characteristics of the UAV. When the angle of attack was small, a trend evincing a wide range of down wash was found on the lower surface of the trailing edge of the wings, reducing the lift coefficient. With increase of angle of attack, this trend weakened, thus increasing the lift coefficient. The changes in take-off velocity did not significantly influence the longitudinal aerodynamic characteristics of the UAV. With increasing take-off velocity, the pressures on the upper and lower surfaces of the UAV both reduced, but only slightly.

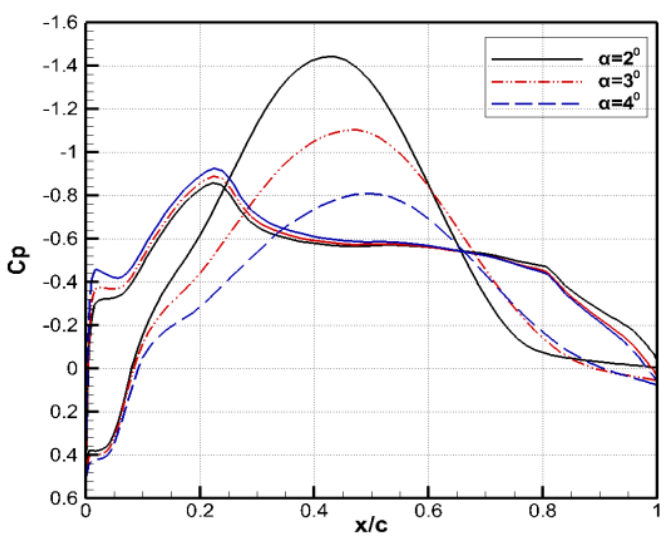

a

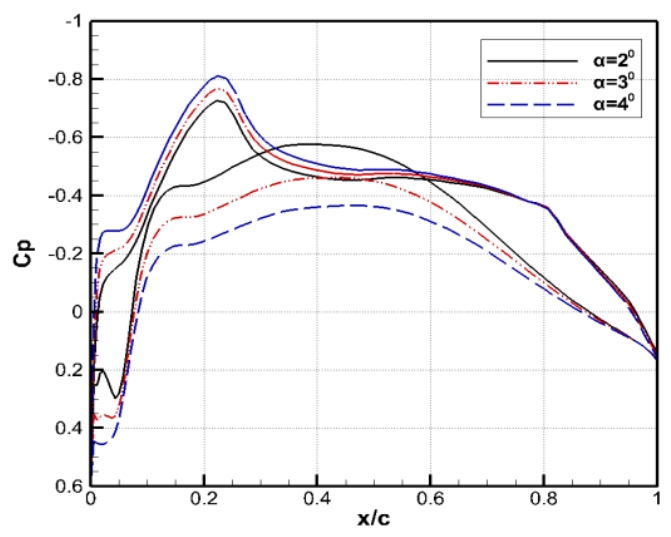

b

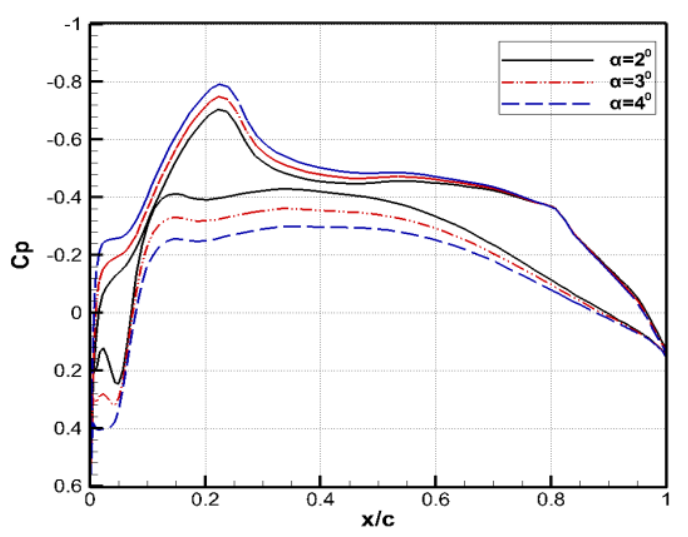

c

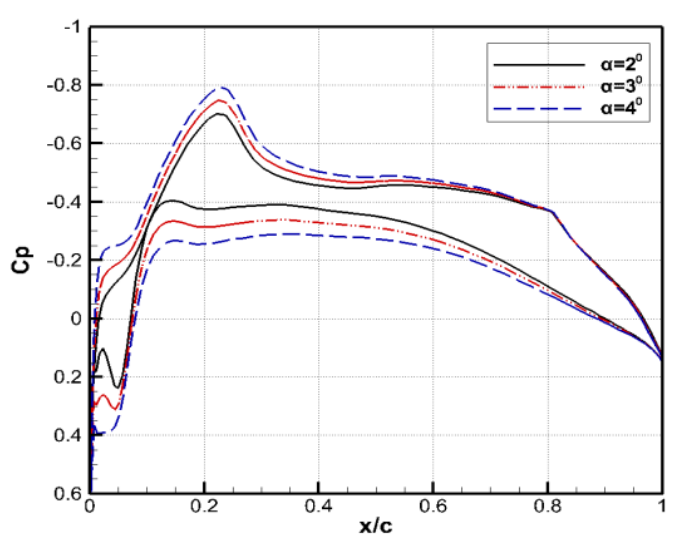

d

Fig. 10 Pressure coefficient distribution of model span wise section at $y=2 \mathrm{~m}$ : (a) $h / c_{A}=0.067$; (b) $h / c_{A}=0.153$; (c); $h / c_{A}=0.235 ;$ (d) $h / c_{A}=0.329$ 
3. In this research on a UAV with medium-aspectratio flying wing configuration, due to the strong suction force generated on the lower surface of the wings, the lift coefficient and the pitching stability decreased with the height of the ground decreased. This differed from the ground effects seen in ground-effect vehicles. In fact, the magnitude of the suction force acting on the lower surface of the wings was affected by the geometric shape of aerofoil to a significant extent, therefore, not all medium-aspect-ratio UAVs have a decreasing lift coefficient at lower height of the ground.

\section{Acknowledgements}

This work was supported by the National Natural Science Foundation of china (No: 51605270), the Science Technology Department Key Project of Shaanxi Province (No: 2017ZDXM-GY-138), the Natural Science Research Project of Shaanxi Province (No: 2016JM1030) and Shaanxi Provincial Department of Education Scientific Research Project (15JK1136).

\section{References}

1. Zhao, X. 1999. Research of ground effect influence on take-off of supersonic pilotless aircraft, Aviation Engineerging \& Mainienance: 43-44.

https://doi.org/10.3969/j.issn.16720989.1999.01.018.

2. Gong, J.F.; Zhu, X.P.; Zhou, Z. 2012. Research on ground effect of unmanned aerial vehicle based on dynamic structured grid, Advances in Aeronautical Science and Engineering 3: 263-268.

https://doi.org/10.3969/j.issn.1674-8190.2012.03.003.

3. Qu, Q.; Jia, X.; Wang, W.; Liu, P.; Agarwal, R. K. 2014. Numerical study of the aerodynamics of a NACA 4412 airfoil in dynamic ground effect, Aerospace Science and Technology 38: 56-63. https://doi.org/10.1016/j.ast.2014.07.016.

4. Ahmed, M. R.; Sharma, S. D. 2005. An investigation on the aerodynamics of a symmetrical airfoil in ground effect, Experimental Thermal and Fluid Science 29: 633647. https://doi.org/10.1016/j.expthermflusci.2004.09.001.

5. Ahmed, M. R.; Takasaki, T.; Kohama, Y. 2007. Aerodynamics of a NACA4412 Airfoil in Ground Effect, AIAA Journal 45: 37-47. https://doi.org/10.2514/1.23872.

6. Coulliette, C.; Plotkin, A. 1995. Airfoil ground effect revisited, AIAA Paper 95-1832: 562-576. https://doi.org/10.2514/6.1995-1832.

7. Buell, D.A.; Tinling, B.E.1957. Ground effects on the longitudinal characteristics of two models with wings having low aspect ratio and pointed tips, NASA TN4044.

8. Fink, M. P.; Lastinger, J. L.1961. Aerodynamic characteristics of low-aspect-ratio wings in close proximity to the ground, NASA TN D-926.

9. Paulson, J. W.; Kjelgaard, S. O.1982. An experimental and theoretical investigation of thick wings at various sweep angles in and out of ground effect, NASA TP-2068.

10. Kjelgaard, S. O.; Paulson, J. W. J. 1980. Effect of sweep and aspect ratio on the longitudinal aerodynamics of a spanloader wing in and out of ground effect, NASA TM-80199.

11. Zerihan, J.; Zhang, X. 2000. Aerodynamics of a single element wing in ground effect, Journal of Aircraft 37: 1058-1064. https://doi.org/10.2514/2.2711.

12. Zhang, X.; Zerihan, J. 2003. Off-Surface Aerodynamic Measurements of a Wing in Ground Effect, Journal of Aircraft 40: 716-725. https://doi.org/10.2514/2.3150.

13. Corsiglia, V. R.; Koenig, D. G.; Morelli, J. P. 1969. Large-scale tests of an airplane model with a doubledelta wing, including longitudinal and lateral characteristics and ground effects, NASA TN D-5102.

14. Fox, H. C. J. 1969.Prediction of lift and drag for slender sharp-edge delta wings in ground proximity, NASA TN D-4891.

15. Coe, P. L. J.; Thomas, J. L. 1979.Theoretical and experimental investigation of ground-induced effects for a low-aspect-ratio highly swept arrow-wing configuration, NASA TP-1508.

16. Jones, B. L.; Franke, M. E.; Stephen, E. J. 2006. aerodynamic ground effects of a tailless chevron-shaped ucav model, 24th Applied Aerodynamics Conference, San Francisco, California, 5-8 June. https://doi.org/10.2514/6.2006-2832.

17. In, W. 2006. Experimental investigation into the aerodynamic ground effect of a tailless chevron and lambdashaped UCAVs, Ohio: Air Force Institute of Technology, ADA451290.

18. Mostaccio, J. T. 2006. Experimental investigation of the aerodynamic ground effect of a tailless lambdashaped UCAV with wing flaps, Ohio: Air Force Institute of Technology, ADA453970.

19. Yang, M. Q.; Ma, D. L. 2013. Effect of asymmetric ground effect on stability of tailless aircraft, Journal of Aerospace Power 28:2553-2560. https://doi.org/10.13224/j.cnki.jasp.2013.11.021

20. Gaitonde,A. L.; Jones, D. P.; Fiddes, S. P. 2016. A 2D Navier-Stokes method for unsteady compressible flow calculations on moving meshes, Aeronautical Journal 102: 89-97. https://doi.org/10.1017/S000192400006557X.

21. Orang, A. A. 2014. Genuinely characteristic-based scheme for the incompressible turbulent flows, Computers \& Fluids 103: 175-185. https://doi.org/10.1016/j.compfluid.2014.07.015.

22. Song, W. P.; Yang, Y.; Qiao, Z. D.; Pahlke, K. 1999. Unsteady N-S calculations using a dual-time stepping method for airfoil oscillating at high angle of attack, Acta Aerodynamica Sinica 17: 466-471. https://doi.org/10.3969/j.issn.0258-1825.1999.04.016 
Junli Wang, Wensheng Zhang, Bolin Feng, Zhigui Ren, Qinghe Zhao

CHARACTERISTICS OF GROUND EFFECTS OF AN
UNMANNED AERIAL VEHICLE WITH HIGH-
ASPCET-RATIO FLYING WINGS DURING TAKE-OFF

S u m m a r y

In view of the take-off process of an unmanned aerial vehicle (UAV) with medium-aspect-ratio flying wing configurations, the influences of ground effects on longitudinal aerodynamic characteristics of the UAV were investigated. By using a numerical simulation method, the effects of different angles of attack, heights from the ground, and take-off velocity on the UAV were studied. Moreover, based on comparisons with test data in previous literature, the numerical calculation method was verified to be accurate. The height from the ground is the primary factor influencing longitudinal aerodynamic characteristics of the
UAV. With the increase in height from the ground, the lift and drag coefficients of the UAV, and its pitching stability, increased. When the angle of attack was small, a large suction area was generated on the lower surface of wings with a large suction amplitude therewith. This results in a large range of downwash. As the angle of attack increased, the lower surface of the trailing edge for wings presents weakening downwash. As the take-off velocity rose, the suction area on the lower surface of the UAV, and its amplitude, gradually decreased, and the negative pressure on the upper surface also decreased to a certain extent. The research results can provide a certain theoretical basis for the safe design of take-off operations for medium-aspect-ratio UAVs.

Keywords: medium-aspect-ratio UAV, ground effect, dynamic layering, numerical simulation.

Received June 12, 2018

Accepted October 18, 2018 